\section{Características basales, manejo de terapias antitrombóticas y pronóstico de pacientes chilenos con FA no valvular. Lecciones del Registro GARFIELD AF en Chile}

\author{
RAMÓN CORBALÁN ${ }^{1}$, CARLOS CONEJEROS ${ }^{2}$, CARLOS REY ${ }^{3}$, \\ BENJAMÍN STOCKINS ${ }^{4}$, GERMÁN EGGERS ${ }^{5}$, CARLOS ASTUDILLO ${ }^{6}$, \\ FERNANDO LANAS ${ }^{4}$, SERGIO POTTHOFF ${ }^{7}$, CÉSAR HOUZVIC ${ }^{8}$, \\ HUMBERTO MONTECINOS ${ }^{9}$, GUSTAVO CHARME ${ }^{10}$, \\ CLAUDIO BUGUEÑO ${ }^{11}$, JUAN AGUILAR ${ }^{12}$, GERMÁN ARRIAGADA ${ }^{13}$, \\ PATRICIO MARÍN ${ }^{14}$, MARTÍN LARICO ${ }^{15}$, \\ EN REPRESENTACIÓN DEL GRUPO GARFIELD AF
}

\section{Features, management and prognosis of Chilean patients with non valvular atrial fibrillation: GARFIELD AF registry}

Background: Atrial fibrillation (AF) is the most common cardiac arrhythmia and is associated with high rates of death, ischemic stroke and systemic embolism (SE). There is scarce information about clinical characteristics and use of antithrombotic therapies in Chilean patients with non-valvular AF. Aim: To describe the characteristics and 1-year outcomes of patients with recently diagnosed AF recruited in Chile into the prospective global GARFIELD-AF registry. Material and Methods: Between 2011-2016, we prospectively registered information of 971 patients recruited at 15 centers, 85\% of them from the public system and $15 \%$ from the private sector. Demographics, clinical characteristics and use of antithrombotic therapies were recorded for all patients. Adverse clinical outcomes were analyzed in 711 patients with 1-year follow-up. Results: The mean age was 71.5 years (66-79), 50\% were men. Mean CHAD2S2 Vasc and HAS BLED scores for stroke risk were 3.3 (2.0-4.0) and 1.5 (1.0-2.0) respectively. Oral anticoagulants were prescribed in $82 \%$ of patients. Seventy percent received Vitamin K antagonists, $10 \%$ novel direct anticoagulants or antiplatelet therapy and only $8 \%$ did not receive any antithrombotic therapy. Mean time in optimal therapeutic range (an international normalized ratio of 2 to 3 ), was achieved in only 40.7\% (23.0-54.8) of patients receiving Vitamin K antagonists. One year rates of death, stroke/systemic embolism and bleeding were 4.75 (3.36-6.71), 2.40 (1.47-3.92) and 1.64\% (0.91-2.97) per 100 person-years. Ischemic stroke occurred in $1.8 \%$ and hemorrhagic stroke in $0.8 \%$ of patients at 1-year of follow up. Conclusions: Although the use of vitamin Kantagonists at baseline was high, the mean time in optimal therapeutic range was low. Mortality and stroke rates are higher than those reported in other contemporary registries.

(Rev Med Chile 2017; 145: 963-971)

Key words: Anticoagulants; Atrial Fibrillation; Chile; Prognosis.
'División Enfermedades

Cardiovasculares, Escuela de

Medicina, Pontificia Universidad

Católica de Chile. Santiago, Chile.

${ }^{2}$ Hospital Barros Luco Trudeau.

Santiago, Chile.

${ }^{3}$ Hospital del Salvador. Santiago,

Chile.

${ }^{4}$ Universidad de La Frontera Hospital Dr. Hernán Henríquez

Aravena. Temuco, Chile.

${ }^{5}$ Hospital Base de Valdivia. Chile.

${ }^{6}$ Hospital Carlos Van Buren.

Valparaíso, Chile.

${ }^{7}$ Hospital Base de Osorno. Chile.

${ }^{8}$ Clínica Santa Lucía. Santiago,

Chile.

${ }^{9}$ Hospital Sótero del Río.

Santiago, Chile.

${ }^{10} \mathrm{Hospital}$ Naval Almirante Nef.

Valparaíso, Chile.

${ }^{11}$ Hospital San Juan de Dios. La

Serena, Chile.

${ }^{12}$ Hospital Clínico de Magallanes

Dr. Lautaro Navarro Avaria. Punta

Arenas, Chile.

${ }^{13} \mathrm{Hospital}$ Clínico Regional Dr.

Guillermo Grant Benavente.

Concepción, Chile.

${ }^{14} \mathrm{Hospital}$ Regional de Talca.

Chile.

${ }^{15}$ Hospital Padre Alberto Hurtado. Santiago, Chile.

Recibido el 12 de mayo de 2017 , aceptado el 9 de agosto de 2017.

Financiamiento: Este trabajo fue financiado por un grant de investigación irrestricto de Bayer Pharma AG, Berlin, Alemania al Thromboisi Research Institute (TRI) de Londres, Reino Unido.

Correspondencia a:

Dr. Ramón Corbalán

División de Enfermedades Cardiovasculares, Escuela de Medicina. Pontificia Universidad Católica de Chile.

Diagonal Paraguay 362, piso 7.

Santiago, Chile.

corbalan@med.puc.cl 
L a fibrilación auricular (FA) es la arritmia cardiaca sostenida más frecuente en la población adulta y se asocia con un mayor riesgo de mortalidad, accidente cerebrovascular $(\mathrm{ACV})$ y embolia sistémica $(E S)^{1,2}$. Su prevalencia aumenta con la mayor edad y se asocia por lo tanto a comorbilidades que influyen en su pronóstico ${ }^{3}$.

La prevalencia de FA en Latinoamérica es similar a la reportada en grandes estudios epidemiológicos. Se estima que afecta a $1,6 \%$ de la población global, variando desde $0,4 \%$ en edades menores de 50 años hasta $8,26 \%$ sobre la edad de 80 años, con $75 \%$ de casos observados en adultos mayores de 60 años 4

La información sobre las características de pacientes con FA no valvular chilenos es escasa. Se ha reportado como una causa frecuente de hospitalización en la población adulta y que es una de las principales causas de accidente cerebrovascular isquémico $^{5-7}$.

El registro Garfield AF se inició en el 2010 en 35 países del mundo. Se ha registrado en forma prospectiva y sistemática información sobre pacientes con FA no valvular a través de 5 cohortes anuales consecutivas llegando a un total de 55.000 pacientes $^{8,9}$.

Chile se incorporó a este registro a partir de la segunda cohorte con 15 centros. En el presente trabajo reportamos por primera vez las características clínicas, manejo y resultados de las terapias antitrombóticas y los eventos cardiovasculares observados a un año de evolución en pacientes chilenos con FA no valvular.

\section{Métodos}

El diseño del registro Garfield AF ha sido reportado previamente ${ }^{8}$. Se han reclutado en forma secuencial y consecutiva pacientes con FA no valvular de diagnóstico reciente. Se entiende por FA no valvular la que ocurre en pacientes que no tienen valvulopatía previa sean de origen reumático o degenerativas. En Chile han participado 15 centros de distintas zonas del país, cada uno con un investigador y enfermera responsable.

Los criterios de inclusión han sido pacientes mayores de 18 años, hombres o mujeres con diagnóstico de FA reciente (menos de 6 semanas) y que tuvieran al menos un factor de riesgo de ACV de acuerdo al puntaje CHAD2S2 $\mathrm{Vasc}^{10}$. El concepto de nueva FA corresponde a pacientes en los cuales la forma definitiva de FA (paroxística, persistente o permanente) aún no estaba definida y para lo cual se esperó un tiempo prudencial de 4 meses, al momento del primer seguimiento.

Los pacientes fueron reclutados en forma prospectiva en distintos sitios de diagnóstico como servicios de urgencia, hospitales o consultas ambulatorias y seleccionados por diversos profesionales. Se ha registrado información sobre características clínicas basales y demográficas, distribución de factores de riesgo para ACV y sangrado, terapias antitrombóticas utilizadas y eventos médicos adversos cardiovasculares y no cardiovasculares.

El protocolo de registro fue aprobado por los comités de ética institucionales, elaborado siguiendo la declaración de Helsinki y las guías internacionales de buenas prácticas clínicas. El registro no contempló intervenciones terapéuticas adicionales a las prescritas por los médicos tratantes ni modificaciones a la dosificación del anticoagulante. La administración de la terapia se hizo mayoritariamente en clínicas de anticoagulación a cargo de enfermeras especializadas. Cada paciente firmó un consentimiento informado y se mantuvo su anonimato y confidencialidad.

La recolección de la información se efectuó cada 4 meses a través de contactos telefónicos y presenciales hasta completar un período de 24 meses. Los datos fueron recolectados en formato electrónico, almacenados y procesados por un centro coordinador ubicado en Londres, el Thrombosis Research Institute (TRI).

Los datos que se presentan en este análisis fueron extraídos de la base de datos hasta julio de 2016. En cuanto a eventos cardiovasculares se puso especial énfasis en la detección de ACV, embolias sistémicas (ES), hospitalizaciones, mortalidad cardiovascular y no cardiovascular, insuficiencia cardiaca y sangrado.

\section{Análisis estadísticos}

Las variables continuas se expresaron en valores medios, más menos desviación estándar o con medianas y rango intercuartil y las variables categóricas como frecuencia y porcentajes. El uso de terapias antitrombóticas en condiciones basales fue analizado según puntajes de CHAD2S2 Vasc y HAS BLED modificado ${ }^{11}$. 
La ocurrencia de eventos adversos mayores se calculó utilizando el número de eventos, la proporción de pacientes en el centro dividida por la población en riesgo al comienzo del seguimiento, expresándose como tasa de eventos por 100 personas/año, con su intervalo de confianza de $95 \%$. Solo la ocurrencia de un primer evento fue considerada como evento.

Los hazard ratios (HRs) fueron estimados del modelo proporcional de Cox después de imputaciones múltiples por el algoritmo de elecciones en cadena (MICE). Los análisis fueron efectuados en TRI utilizando la versión SAS 9.4.

\section{Resultados}

\section{Casuística}

En Chile, se registró un total de 971 pacientes entre las cohortes 2 y 5 en el período 2011-2014 a través de 15 centros, base sobre la cual se describen las características clínicas y terapias basales. De estos 971 pacientes un total de 711 completaron el primer año de seguimiento y sobre esta base se describen los eventos médicos adversos que se observaron en esta casuística.

\section{Características basales}

Estas se presentan en detalle en la Tabla 1. La edad promedio de nuestra serie fue de 71,5 años y 50,9\% eran hombres. Entre los factores de riesgo cardiovascular los más prevalentes fueron hipertensión arterial $(85,6 \%)$, hipercolesterolemia $(40 \%)$ y obesidad $(40,1 \%)$. Entre las formas de presentación clínica de la FA las más frecuentes fueron: nueva FA $(37,7 \%)$ y FA paroxística $(32,2 \%)$.

El $85 \%$ de los pacientes fueron reclutados en el sector público y $14,7 \%$ en sector privado, predominando las especialidades de Cardiología y Atención Primaria en esta etapa basal (Tabla 2).

El puntaje CHAD2S2 Vasc promedio fue $3,3 \mathrm{y}$ HAS BLED 1,5. Al momento del ingreso 81,9\% de los pacientes fueron tratados con anticoagulante orales con o sin terapia antiplaquetaria asociada, $70 \%$ del total recibieron antagonistas de vitamina $\mathrm{K}$ (AVK), predominantemente Acenocumarol. El 10\% restante nuevos anticoagulantes directos. Sólo 10,3\% fueron tratados con antiplaquetarios y $7,7 \%$ quedaron sin terapia antitrombótica (Tabla 3).
Tabla 1. Características basales en GARFIELD-AF

\begin{tabular}{|c|c|}
\hline Características basales & $\mathbf{n}=971$ \\
\hline Edad, media $\pm S D$, años & $71,5(11,2)$ \\
\hline \multicolumn{2}{|l|}{ Edad, n (\%) } \\
\hline$<65$ & $223(23,0)$ \\
\hline $65-74$ & $311(32,0)$ \\
\hline$\geq 75$ & $437(45,0)$ \\
\hline Género, Masc n (\%) & $494(50,9)$ \\
\hline \multicolumn{2}{|l|}{ Tipo de FA, n \% } \\
\hline Permanente & $98(10,1)$ \\
\hline Persistente & $194(20,0)$ \\
\hline Paroxística & $313(32,2$ \\
\hline Nueva FA & $366(37,7)$ \\
\hline \multicolumn{2}{|l|}{ Factores de riesgo } \\
\hline Hipertensión arterial & $829(85,6)$ \\
\hline Diabetes, n (\%) & $247(25,4)$ \\
\hline Hipercolesterolemia, n (\%) & $355(40,1)$ \\
\hline Tabaco (actual o previo) & $201(21,9)$ \\
\hline Obesidad IMC > 30, n (\%) & $241(40,1)$ \\
\hline \multicolumn{2}{|l|}{ Antecedentes Médicos, n (\%) } \\
\hline Insuficiencia cardíaca & $170(17,5)$ \\
\hline Enfermedad Vascular & $91 \quad(9,4)$ \\
\hline Enfermedad Coronaria & $111(11,4)$ \\
\hline Síndromes Coronarios Agudos & $68(7,0)$ \\
\hline Enfermedad Carotídea & $12(1,2)$ \\
\hline $\mathrm{FE} \mathrm{VI} \geq 40 \%^{c}$ & $336(90,3)$ \\
\hline ACV/TIA & $93(9,6)$ \\
\hline IRC moderada a severa & $61 \quad(6,3)$ \\
\hline Historia de sangrado & $26 \quad(2,7)$ \\
\hline Embolía sistémica & $16(1,7)$ \\
\hline Score $\mathrm{CHA}_{2} \mathrm{DS}_{2}-\mathrm{VASC}$, media $\pm \mathrm{SD}$ & $3,3(1,5)$ \\
\hline Score HAS-BLED, media \pm SD & $1,5(0,8)$ \\
\hline
\end{tabular}

Abreviaciones: IMC = índice masa corporal; IRC = insuficiencia renal crónica; $\mathrm{SD}=$ desviación estándar; $\mathrm{TI} A=$ accidente vascular transitorio.

Tabla 2. Sectores de tratamiento y especialidades médicas

n $(\%)$

$\begin{array}{ll}\text { Tratamiento en sector } & \\ \text { Público } & 821(85,3) \\ \text { Privado } & 142(14,7)\end{array}$

Especialidades médicas al momento

del ingreso

Cardiología

$464(48,2)$

Medicina Interna

$256(26,4)$

Neurología

$15(1,5)$

Atención primaria 
Tabla 3. Terapias antitrombóticas al ingreso

\begin{tabular}{|lc|}
\hline & $\mathbf{n}=\mathbf{9 5 1}$ \\
\hline $\mathrm{AVK}, \%$ & $519(54,0)$ \\
$\mathrm{AVK}+\mathrm{AP}, \%$ & $156(16,2)$ \\
$\mathrm{FXa}, \%$ & $61(6,3)$ \\
$\mathrm{FXa}+\mathrm{AP}, \%$ & $31(3,2)$ \\
$\mathrm{IDT}, \%$ & $16(1,7)$ \\
$\mathrm{IDT}+\mathrm{AP}, \%$ & $5(0,5)$ \\
AP, \% * & $99(10,3)$ \\
Sin tratamiento & $74(7,7)$ \\
\hline
\end{tabular}

Abreviaturas: AVK = Antagonistas de vitamina $K ; F X a=I n-$ hibidor de Factor XA; IDT = Inhibidor Directo de Trombina; $\mathrm{AP}=$ Antiplaquetarios.

En las Figuras 1 y 2 se muestra la distribución de terapias antitrombóticas según los puntajes CHADs2 Vasc y HAS BLED y se comparan con los utilizados en el resto del mundo. La proporción de pacientes que reciben terapia anticoagulante oral aumentó progresivamente desde el puntaje CHADs Vasc 3 y se mantuvo posteriormente estable (Figura 3). A la inversa el empleo de antiplaquetarios aumentó con el incremento del puntaje HAS BLED. En la Figura 4 se muestra la proporción de pacientes que recibieron terapias antitrombóticas en las cohortes 2 y 3 , comparando con las del resto del mundo. Se puede apreciar que la distribución de pacientes tratados con AVK en
Chile se mantuvo estable, mientras en el resto del mundo aumentó la indicación de anticoagulantes orales directos.

Se registraron 3.849 lecturas de INR y solo $35,6 \%$ tuvo promedios entre 2 y 3 . El tiempo en rango terapéutico (TRT), se calculó con el método de Rosendal ${ }^{12}$ sobre una base de 327 pacientes, observándose una mediana de 40,7\% (Tabla 4).

\section{Eventos médicos adversos}

En la Tabla 5 se muestra la distribución de eventos médicos adversos. Las tasas de mortalidad global, ACV/ES y sangrado fueron de 4,75, 2,40 y 1,64 por 100 años/persona respectivamente. La

Tabla 4. Distribución de valores de INR y proporción de tiempo en rango terapéutico (TTR) en pacientes con AVK al ingreso

\begin{tabular}{|lc|}
\hline INR, N $N^{\circ}$ de lecturas & 3.849 \\
\hline INR, mediana (IQR) & $2,0(1,6$ a 2,6$)$ \\
\hline $\begin{array}{l}\text { INR, No de lecturas }(\%) \\
\quad<2,0\end{array}$ \\
$2,0-3,0$ & $1.930(50,1)$ \\
$>3,0$ & $1.369(35,6)$ \\
TTR, No & $550(14,3)$ \\
TTR, mediana (IQR) & 327 \\
\hline
\end{tabular}

Abreviaturas: INR = international normalized ratio. Nota: Los valores de INR se presentan como número del total de lecturas y porcentaje.

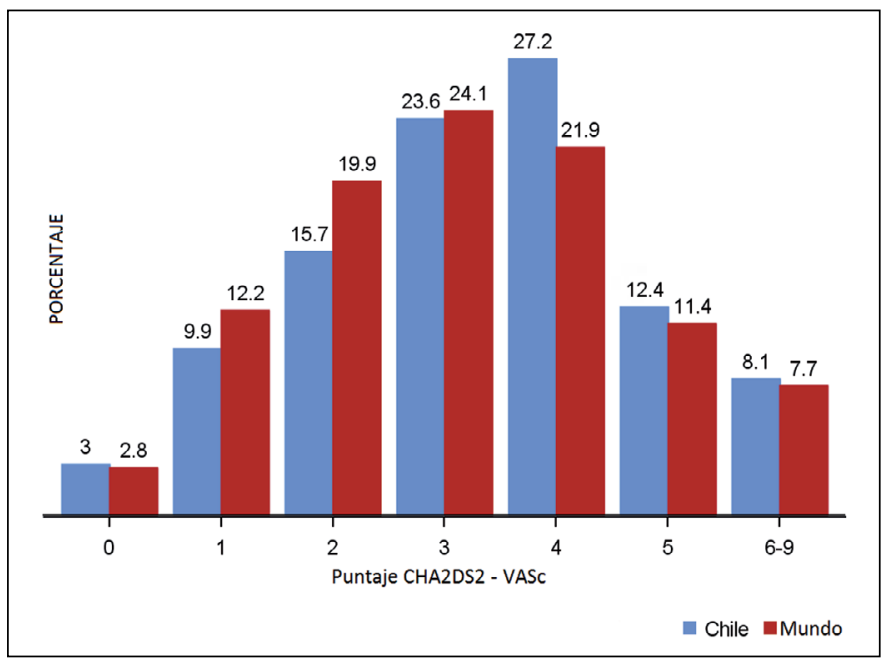

Figura 1. Perfil de riesgo de $A C V$ según puntaje CHA2DS2-VASC score en la población chilena comparada con el resto del mundo. 

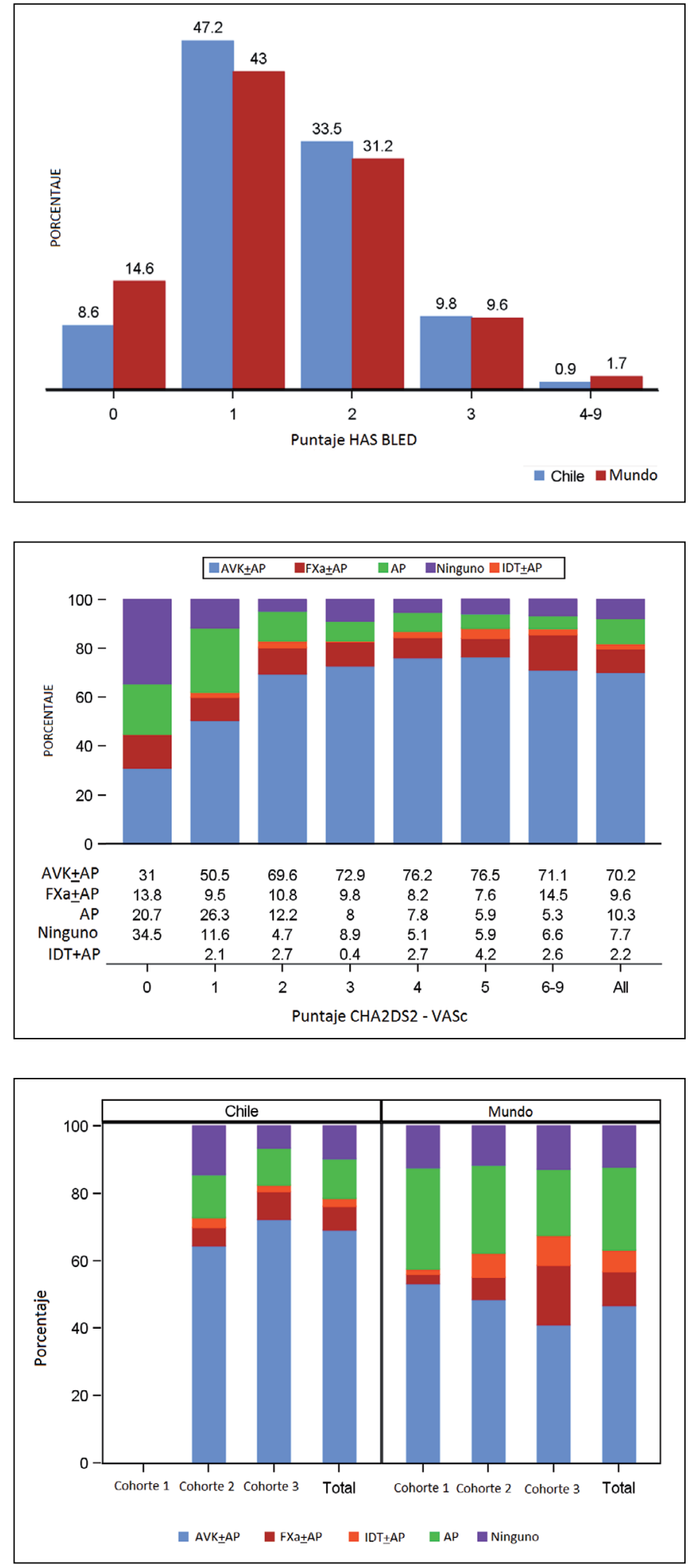

Figura 2. Perfil de riesgo de sangrado según puntaje HAS BLED en la población chilena comparada con el resto del mundo.
Figura 3. Empleo de terapias antitrombóticas basales según puntaje de CHAD2S2 Vasc. AVK + $\mathrm{AP}=$ antagonistas de vitamina $\mathrm{K}$ y antiplaquetarios; $F X a+A P=$ inhibidores factor $X a$ y antiplaquetarios; $A P=$ antiplaquetarios; IDT $+A P=I n-$ hibidores directos de trombina y antiplaquetarios.

Figura 4. Evolución de terapias antitrombóticas basales en las distintas cohortes del registro en Chile y resto del mundo. $\mathrm{AVK}+\mathrm{AP}=$ antagonistas de vitamina $K$ y antiplaquetarios; $F X a+A P=$ in hibidores factor $\mathrm{Xa}$ y antiplaquetarios; $\mathrm{AP}=$ antiplaquetarios; IDT $+\mathrm{AP}=$ Inhibidores directos de trombina y antiplaquetarios. 
Tabla 5. Tasas de eventos durante el primer año de evolución en Cohortes 1 a 4; $n=711$ pts

\begin{tabular}{|c|c|c|c|c|}
\hline Evento & Causa & $\begin{array}{c}n \\
\text { eventos }\end{array}$ & $\begin{array}{c}\text { Tasa eventos/100 años } \\
\text { persona } \\
\mathbf{p} \\
\text { personas/año }\end{array}$ & $95 \%$ IC \\
\hline Mortalidad global & $\begin{array}{l}\text { Muertes cardiovasculares } \\
\text { Muertes no-cardiovasculares } \\
\text { Causa indeterminada }\end{array}$ & $\begin{array}{r}32 \\
17 \\
12 \\
3\end{array}$ & $\begin{array}{l}4,75 \\
2,52 \\
1,78 \\
0,45\end{array}$ & $\begin{array}{l}(3,36 \text { a } 6,71) \\
(1,57 \text { a } 4,06) \\
(1,01 \text { a } 3,14) \\
(0,14 \text { a } 1,38)\end{array}$ \\
\hline ACV/ES & & 16 & 2,40 & $(1,47$ a 3,92$)$ \\
\hline Sangrado mayor & & 11 & 1,64 & $(0,91$ a 2,97$)$ \\
\hline Síndrome coronario agudo & & 5 & 0,74 & $(0,31$ a 1,78$)$ \\
\hline Insuficiencia cardíaca & & 5 & 0,75 & $(0,31$ a 1,79$)$ \\
\hline Causas de muerte & & & & \\
\hline Cardiovasculares & $\begin{array}{l}\text { Infarto al miocardio } \\
\text { Insuficiencia cardíaca } \\
\text { Muerte súbita } \\
\text { Otra }\end{array}$ & $\begin{array}{l}17 \\
17 \\
17 \\
17\end{array}$ & $\begin{array}{l}1 \\
4 \\
3 \\
9\end{array}$ & $\begin{array}{r}5,88 \\
23,53 \\
17,65 \\
52,94\end{array}$ \\
\hline No cardiovasculares & $\begin{array}{l}\text { Insuficiencia respiratoria } \\
\text { Infección/sepsis } \\
\text { Tumor maligno } \\
\text { Otra }\end{array}$ & $\begin{array}{l}12 \\
12 \\
12 \\
12\end{array}$ & $\begin{array}{l}2 \\
2 \\
3 \\
5\end{array}$ & $\begin{array}{l}16,67 \\
16,67 \\
25,00 \\
41,67\end{array}$ \\
\hline
\end{tabular}

tasa de mortalidad cardiovascular fue 2,52 y no cardiovascular de 1,78\% por 100 años/persona.

Entre las causas de muerte cardiovascular destacan la insuficiencia cardiaca $(23,5 \%)$ y la muerte súbita $(17,6 \%)$. Entre las causas de muerte no cardiovascular predominan los tumores malignos (25\%).

Los ACV isquémicos con o sin complicación hemorrágica se observaron en $1,4 \%$ de la población y los ACV hemorrágicos en $0,8 \%$ (Tabla 6).

Tabla 6. Tipo de ACV durante el primer año de evolución. Cohortes 1 a 4

\begin{tabular}{|lc|}
\hline Tipo de ACV & n (\%) \\
\hline ACV (no incluyendo embolía sistémica) & $14(1,97)$ \\
\hline ACV Isquémico & $8(1,13)$ \\
Con conversión hemorrágica & $2(0,28)$ \\
\hline ACV hemorrágico & $6(0,84)$ \\
Intracerebral & $4(0,56)$ \\
Subaracnoideo & $1(0,14)$ \\
Intraventricular & $1(0,14)$ \\
\hline
\end{tabular}

\section{Discusión}

\section{Características basales}

Esta serie chilena del registro Garfield AF es una muestra representativa de nuestra realidad, ya que participaron 15 centros y $85 \%$ de ellos eran del sector público y solo $15 \%$ del sector privado. Igualmente destaca el que las características basales de nuestros pacientes son muy similares a las reportadas por el mismo Registro Garfield AF a nivel mundial y por otras grandes series clínicas ${ }^{10,13,14}$. La edad promedio fue alrededor de 70 años y la proporción de hombres y mujeres fue similar. Asimismo la distribución de pacientes según puntajes de riesgo $\mathrm{CHAD} 2 \mathrm{~S} 2$ Vasc y HAS BLED fue muy similar a la de grandes series. Las formas de presentación clínica de la FA más frecuentes fueron nueva FA y FA paroxística.

\section{Terapias antitrombóticas}

En la casuística chilena llama la atención la alta tasa de empleo de terapia anticoagulante oral, fundamentalmente a base de AVK desde el 
inicio de la participación en el registro, la cual fue superior a las reportadas en distinto registros contemporáneos ${ }^{15-17}$. El empleo de nuevos anticoagulantes directos no superó $10 \%$ y se mantuvo constante a lo largo de los años, reflejando su falta de disponibilidad en el sistema público, así como su costo aún elevado. Llama la atención que el INR entre 2 y 3 se registró solo en $35 \%$ de los pacientes y que el tiempo en rango terapéutico (TRT) alcanzó una mediana de $40 \%$, muy inferior al recomendado en las guías clínicas internacionales ${ }^{18,19}$.

En una publicación reciente de Haas y cols. sobre el rol pronóstico del TRT en pacientes tratados con AVK se observó que las tasas de ACV/ ES y sangrado fueron significativamente menores en los pacientes que alcanzaban un TRT mayor de $65 \%$, lo que se traducía además en menor mortalidad ${ }^{20}$. Resultados similares habían sido reportados en otra serie clínica por Hylek y cols. ${ }^{21}$. En el registro español Califa se reportó también un control inadecuado del TRT, pero con cifras promedio de $50 \%{ }^{22}$. En el ensayo clínico RE-LY también se comunicó una gran variación de rangos de INR según países y centros ${ }^{23}$. Lo mismo ha sido reportado en otras series clínicas contemporáneas, lo que refleja un problema universal con el manejo de los $\mathrm{AVK}^{24-26}$.

Podría especularse que en nuestro medio influyen una serie de variables relacionadas con la educación y adhesión de los pacientes, rotación de empleo de fármacos genéricos en el sistema público, rotación de los profesionales de la salud encargados del control del tratamiento anticoagulante, variaciones influidas por cambios en la dieta o empleo de otros fármacos, etc. En todo caso estos resultados debieran constituir un llamado de alerta sobre la necesidad de mejorar políticas públicas para un mejor control de la terapia anticoagulante con AVK. En nuestro medio el porcentaje de empleo de nuevos anticoagulantes directos se ha mantenido alrededor de $10 \%$ en los 4 años de participación en Garfield AF, a diferencia de lo observado en la gran mayoría de países que participan en este registro, donde se observa un incremento progresivo de estos fármacos cuyo perfil de seguridad es mayor ${ }^{27,28}$.

\section{Eventos médicos adversos}

Nuestros resultados muestran que los principales eventos médicos adversos son la morta- lidad (cardiovascular y no cardiovascular), el ACV y el sangrado mayor en tasas de 4,$75 ; 2,4$ y 1,64 por 100 años/persona respectivamente. Estos datos confirman observaciones previas del registro Garfield AF en los que se encontró que la mortalidad a 2 años de seguimiento casi triplicaba la incidencia de $\mathrm{ACV}^{29}$. A diferencia de lo comunicado por Bassand y cols en nuestro medio las tasas de mortalidad y ACV al año de seguimiento son superiores a las encontradas en el estudio global a 2 años, lo que refleja la complejidad del manejo de la FA. Más allá de la prevención de ACV y sangrado la alta mortalidad representa el principal desafío para el tratamiento de esta arritmia y ello implica la necesidad de un adecuado tratamiento no solo antitrombótico sino que también de las comorbilidades asociadas como insuficiencia cardíaca, insuficiencia renal, diabetes y otras. De hecho la principal causa de muerte cardiovascular fue la insuficiencia cardíaca, similar a lo reportado por Bassand y cols y a los resultados del subestudio Engage para Latinoamérica $^{29,30}$. Las razones para las mayores tasas de mortalidad y ACV en Chile pueden ser multifactoriales y representan un desafío para mejorar el tratamiento de la FA, que constituye más un síndrome que una arritmia cardíaca aislada. En nuestro medio se observa que la proporción de ACV hemorrágico es alta, lo que puede estar en concordancia con un porcentaje alto $(45 \%)$ de pacientes mayores de 75 años o que por la existencia de comorbilidades pudieran tener mayor riesgo de sangrado. En resumen, las características basales de pacientes chilenos con FA son similares a las reportadas universalmente. Se observa una alta tasa de empleo de AVK para la prevención de ACV, pese a lo cual el control de esta terapia, evaluado por el TRT, es deficiente y se asocia a una mayor tasa de ACV isquémicos y hemorrágicos, Se confirma, además, que los pacientes con FA no valvular tienen una alta tasa de mortalidad cardiovascular y no cardiovascular, lo que representa un desafío adicional para su enfoque terapéutico.

Agradecimientos. Agradecemos a los médicos, enfermeras y pacientes involucrados en el Registro Garfield AF, a Emily Chu y Gloria Kayani por su apoyo editorial, a Gabriele Acceta por su apoyo estadístico y al Comité Ejecutivo del Registro Garfield AF liderado por Lord AJ Kakkar. 


\section{Referencias}

1. Kannel WB, Abbot RD, Savage DD, McNamara PM. Epidemiologic features of chronic atrial fibrillation: the Framingham Study. N Engl J Med 1982; 306: 1018-22.

2. Lubitz SA, Moser C, Sullivan L, Rienstra M, Fontes JD, Villalon ML, et al. Atrial fibrillation patterns and risks of subsequent stroke, heart failure, or death in the community. J Am Heart Assoc 2013; 2: e000126.

3. Schnabel RB, Yin X, Gona P, Larson MG, Beiser AS, Mc manus D, et al. 50 year trends in atrial fibrillation prevalence, incidence, risk factors and mortality in the Framingham Heart Study. Lancet 2015; 386: 154-62.

4. Cubillos L, Haddad A, Kuznitz A, Mould-Quevedo J. Burden of disease from atrial fibrillation in adults from seven countries in Latin America. Int J of Gen Med 2014; 7: 441-8.

5. Corbalán R, Tapia J, Braun S, Arriagada D, Huete I, Valdés F, et al. Estudio multidisciplinario del Accidente Vascula Cerebral: II Perfil cardiovascular de las enfermedades oclusivas. Rev Med Chile 1994; 122: 1021-30.

6. Ortiz M, Morris R, Asenjo R, Cereceda M. Hospitalizaciones asociadas al diagnóstico de Fibrilación Auricular en Chile. Tendencia creciente en la última década. Rev Chil Cardiol 2011; 30: 193-7.

7. Mellado P, Court J, Godoy J, Mery C, Bamett C, Andresen $\mathrm{M}$, et al. Características de la enfermedad cerebrovascular en un Servicio de Cuidados Intermedios Neurológicos, en Chile. Análisis de 459 pacientes consecutivos. Rev Med Chile 2005; 133: 1274-84.

8. Kakkar AK, Mueller I, Bassand JP, Fitzmaurice DA, Goldhaber SZ, Goto S, et al. International longitudinal registry of patients with atrial fibrillation at risk of stroke: Global anticoagulant registry in the field (GARFIELD) Am Heart J 2012; 01(163): 13-9.e1.

9. Kakkar AK, Mueller I, Bassand JP, Fitzmaurice DA, Goldhaber SZ, Goto S, et al. Risk profiles and antithrombotic treatment of patients newly diagnosed atrial fibrillation at risk of stroke Perspectives from the international, observational, prospective GARFIELD registry Plos One 2013; 8 e63479, doc 101371.

10. Lip GY, Niewlaat R, Pisters R, Lane DA, Crijns HJ. Refining clinical risk stratification for predicting stroke and thromboembolism in atrial fibrillation using a novel risk factor-based approach the Euro Heart Survey on Atrial Fibrillation Chest 2010; 137: 263-72.

11. Oisters R, Lane DA, Niewlaat R, de Vos CD, Crijns HJ, Lip GY. A novel user-friendly score (HAS-BLED) to asess one-year risk of major bleeding in atrial fibrillation patients. Chest 2010; 138: 1093-100.

12. Rosendaal F, Camejiester S, Van der Meer F, Briet E. A method to determine the optimal intensity of oral anticoagulant therapy. Thromb Haemost 1993; 69: 236-9.

13. Lip GY, Laroche C, Georghe-Andre D, Santini M, Karlams Z, Vilsted L, et al. "Real world" antithrombotic treatment in atrial fibrillation: The EORP-AF pilot survey. The Am J Med 2014; 127: 519-29.

14. Wilke F, Groth A, Mueller S, Pfannkuche M, Verheyer F, Linder R, et al. Oral anticoagulation use by patients with atrial fibrillation in Germany. Thromb Haemost 2012; 107: 1053-65.

15. Hart RG, Benavente O, Mc Bride R, Pierce LA. Antithrombotic therapy to prevent stroke in patients with atrial fibrillation: a metaanalysis. Ann Intern Med 1999; 131: 492-501.

16. Lip GY, Lane DA, Stroke prevention in atrial fibrillation: A systematic review. JAMA 2015; 313: 1950-62.

17. Piccini JP, Frank ES, Ansell JE, Fonarow GC, Gersh BJ, Go AS, et al Outcomes registry for better informed treatmente of atrial fibrillation: rationale and design of the ORBIT AF. Am Heart J 2011; 162: 606-12.

18. Kirchhof P, Benussi S, Kotecha D, Ahlsson A, Atar D, Casadei B, et al. 2016 ESC Guidelines for the management of atrial fibrillation developed in collaboration with EACTS. Eur Heart J 2016; 37: 2893-962.

19. January CT, Wann LS, Alpert JS, Calkins H, Cigarroa JE, Cleveland JC Jr, et al, ACC/AHA Task Force Members. 2014 AHA/ACC/HRS guidelines for the management of patients with atrial fibrillation: executive summary: a report of the American College of Cardiology/American Heart Association Task Force on practice guidelines and the Heart Rhythm Society. Circulation 2014; 130: 2071 104.

20. Haas S, ten Cate H, Accetta G, Bassand JP, Camm J, Corbalán R, et al. Quality of Vitamin K antagonist control and 1-year outcomes in patients with atrial fibrillation: a global perspective from the GARFIELD AF registry. Plos One 2016; 11: e 01640, doi 10371.

21. Hylek EM, Go AS, Chang Y, Jensvold NG, Hemant LE, Selby JV, et al. Effect of intensity of oral anticoagulation on stroke severity and mortality in atrial fibrillation. $\mathrm{N}$ Engl J of Med 2003; 349: 1010-26.

22. Anguita M, Bertomeu V, Cequier-Fillat R, CALIFA study researchers. Quality of Vitamin K antagonist anticoagulation in Spain: prevalence of poor control and associated factors. Rev Esp Cardiol 2015; 68: 761-8.

23. Van Spall HG, Wallentin L, Yusuf S, Eikelboom JW, Niewlaat R, Yang S, et al. Variation in warfarin dose adjustment practice is responsible for differences in the quality of anticoagulation control between centers and countries: an analysis of patients receiving warfarin in the RE-LY trial. Circulation 2012; 126: 2309-16. 
24. Piccini JP, Hellkamp AS, Lokhnygina Y, Patel MR, Hanell FE, Singer DE, et al. Relationship between time in therapeutic range and comparative treatment effect of rivaroxaban and warfarin: Results from the ROCKET AF trial. J Am Heart Assoc 2014: 3: e 000521, doi 101161.

25. Oldgren J, Healey JS, Ezekowitz M, Commerford P, Avezum A, Prem Pais, et al, on behalf of the RE-LY Atrial Fibrillation Registry Investigators. Variations in cause and management of AF in a prospective registry of 15400 Emergency Department patients in 46 countries. Circulation 2014; 129: 1568-76.

26. Gallego P, Roldán V, Marín F, Romera M, Valdés M, Vicente $\mathrm{V}$, et al. Cessation of oral anticoagulation in relation to mortality and the risks of thrombotic events in patients with atrial fibrillation. Thromb and Haemost 2013; 110: 1189-98.

27. Ruff CT, Giugliano RP, Braunwald E, Hoffman EB,
Deenadayalu N, Ezekowitz MD, et al. Comparison of the efficacy and safety of new oral anticoagulants with warfarin in patients with atrial fibrillation: a meta-analysis of randomised trials. Lancet 2014; 383: 955-62.

28. Camm J, Accetta G, Ambrosio G, Atar D, Bassand JP, Berge E, et al. Evolving antithrombotic treatment patterns for patients with newly diagnosed atrial fibrillation, GARFIELD AF investigators. Heart 2017; 103: 307-14.

29. Bassand JP, Accetta, Camm AJ, Cools F, Fitzmaurice DA, Fox KA, et al. Two-year outcomes of patients with newly diagnosed atrial fibrillation: results from GARFIELD AF. Eur Heat J 2016; 37: 2882-9.

30. Corbalán R, Nicolau JC, López Sendon J, García Castillo A, Botero R, Sotomayor G, et al. Clinical Outcomes with edoxaban vs Warfarin in Latin American Patients with AF. Insights from the ENGAGE AF-TIMI 48 trial. Circulation 2016; 134: A16898. 